\title{
$q$-Fibonacci polynomials and the Rogers-Ramanujan identities
}

\author{
Johann Cigler
}

Fakultät für Mathematik

Universität Wien

A-1090 Wien

Nordbergstrasse 15

Johann.Cigler@univie.ac.at

AMS Subject Classification: 05A19, 05A30, 11B39, 11B65, 11P81

\begin{abstract}
We derive some formulas for the Carlitz $q$-Fibonacci polynomials $F_{n}(t)$ which reduce to the finite version of the Rogers-Ramanujan identities obtained by I. Schur for $t=1$. Our starting point is a representation of the $q-$ Fibonacci polynomials as the weight of certain lattice paths in $\mathbb{R}^{2}$ contained in a strip along the $x$-axis. We give an elementary combinatorial proof by using only the principle of inclusion-exclusion and some standard facts from $q$-analysis.

Keywords: Rogers-Ramanujan identities, $q$-analogue, $q$-Fibonacci polynomial, lattice path, inclusion-exclusion
\end{abstract}

\section{Background}

The Rogers-Ramanujan identities play an important role in the theory of partitions (cf. [3]). They have an unusual history. They were first found by L.J. Rogers in 1894 but remained unnoticed for some time. About 1913 Srinivasa Ramanujan rediscovered the formulas but he had no proof. In 1917 Ramanujan came accidentally across Rogers' paper. He and Rogers then found simpler proofs. At about the same time, I. Schur [18] independently rediscovered the identities and gave two proofs which differed radically from these other proofs. Some new ideas emerged as these identities occurred in mathematical physics in the course of the solution of the so called hard hexagon model by R.J. Baxter in 1981. Today there are plenty of different proofs of these identities and their generalizations. I only want to mention 
some papers which in some way have inspired me in writing this paper: 1, [4], [5], [6], [12], [13], [14], [15], [16], [17], and [18]. It appears that the Rogers-Ramanujan identities have connexions with various different parts of mathematics. An especially interesting aspect is their relation with the Fibonacci numbers. This has first occurred in the paper [18] by I. Schur and emerged again unexpectedly in the solution of the hard hexagon model (cf. [12]). It turned out that the Rogers-Ramanujan identities are q-analogues of identities for the Fibonacci numbers. The common mathematical root of these identities is their interpretation as numbers, respectively weights, of certain sets of lattice paths in $\mathbb{R}^{2}$ contained in a strip along the $x$-axis, which immediately suggests using the principle of inclusion-exclusion. In this paper we give an elementary exposition using only standard facts from $q$-analysis of this aspect of the Rogers-Ramanujan identities together with a slight generalization for $q$-Fibonacci polynomials, which also in the special case $q=1$ seems to be new.

\section{$2 q$-Fibonacci polynomials}

We define the (Carlitz-) $q$-Fibonacci polynomials $([8])$ by the recursion

$$
F_{n}(t)=F_{n-1}(t)+q^{n-3} t F_{n-2}(t)
$$

with initial values

$$
F_{0}(t)=0, F_{1}(t)=1 .
$$

There are two combinatorial models consisting of certain lattice paths in $\mathbb{R}^{2}$ where these polynomials appear in a natural way. First we consider lattice paths of finite length, where at each step only three moves are allowed, a northeast move $(i, j) \rightarrow(i+1, j+1)$, a southeast move $(i, j) \rightarrow(i+1, j-1)$, and a horizontal move $(i, 0) \rightarrow(i+1,0)$ (cf. [1], [6]). Define a peak as a vertex preceded by a northeast step and followed by a southeast step, and a valley as a vertex preceded by a southeast step and followed by a northeast step. The height of a vertex is its $y$-coordinate. The weight of a vertex is $q^{m} t$ where $m$ is its $x$-coordinate. The weight of a path is the product of the weight of its peaks.

Consider now the set of all non-negative lattice paths starting at $(0,0)$ and ending in $(n, 0)$ with maximal height 1 . They consist only of horizontal steps and northeast steps followed by a southeast step. Let $w_{n}(t)$ be their weight. If we consider the initial points of the path we get the recursion

$$
w_{n}(t)=w_{n-1}(q t)+q t w_{n-2}\left(q^{2} t\right),
$$


if we consider the end points of the path we get

$$
w_{n}(t)=w_{n-1}(t)+q^{n-1} t w_{n-2}(t) .
$$

The initial values are in either case

$$
w_{0}(t)=w_{1}(t)=1
$$

This implies that

$$
w_{n}(t)=F_{n+1}(q t) .
$$

If we identify a horizontal step with a dot $\cdot$ and a peak with a dash - then we get the Morse code representation studied in [9].

It is well known (cf. e.g. [8], [9]) that

$$
F_{n+1}(q t)=\sum_{k<n} q^{k^{2}}\left[\begin{array}{c}
n-k \\
k
\end{array}\right] t^{k}
$$

where $\left[\begin{array}{l}n \\ k\end{array}\right]$ denotes the $q$-binomial coefficient. In order to write formulas such as (2.7) as a sum over all integers we assume in this paper that $\left[\begin{array}{l}n \\ k\end{array}\right]=0$ if $n<0$.

Formula (2.7) can also be shown in the following way (cf. Bressoud [6]): The factor $\left[\begin{array}{c}n-k \\ k\end{array}\right]$ is the generating function for partitions into at most $k$ parts or equivalently into exactly $k$ parts where zeroes are permitted, each of which is $\leq n-2 k$ (cf. [3]). Denote these parts by $a_{1} \geq a_{2} \geq \cdots \geq a_{k} \geq 0$. Consider now the unique path of length $2 k$ with $k$ peaks. Its weight is $q^{k^{2}}=q^{1+3+\cdots+(2 k-1)}$. We insert now $a_{k}$ horizontal steps in front of the northeast step of the first peak of this path and $a_{j}-a_{j+1}$ horizontal steps in front of the northeast step of the $(k-j+1)$-th peak. In order to get a path of length $n$ we insert after the last southeast step $n-2 k-a_{1}$ horizontal steps. The weight of this path is then $q^{a_{k}+1+a_{k-1}+3+\cdots+a_{1}+(2 k-1)}$.

Our aim is to show that $F_{n+1}(q t)$ can also be written in the form

$$
F_{n+1}(q t)=\sum_{i \in \mathbb{Z}}(-1)^{i} f_{i}(n, t),
$$

where

$$
f_{i}(n, t)=q^{\frac{i(5 i-1)}{2}} \sum_{s \geq|i|} q^{(s-i)(s+i)}\left[\begin{array}{c}
\left\lfloor\frac{n+i}{2}\right\rfloor \\
s-i
\end{array}\right]\left[\begin{array}{c}
\left\lfloor\frac{n-i+1}{2}\right\rfloor \\
s+i
\end{array}\right] t^{s} .
$$


We will show this by using an inclusion-exclusion argument. To this end we introduce another lattice path model (cf. [12]):

To each non-negative lattice path $u$ with maximal height 1 starting at $(0,0)$ and ending in $(n, 0)$ we associate a lattice path $T u$ in the strip $-2 \leq y \leq 1$ without horizontal steps starting at $(0,0)$ and ending in $(n, 0)$ or $(n,-1)$ such that each horizontal step is transformed into a step whose vertices lie on the lines $y=0$ and $y=-1$ and where the peaks are either unaffected or are changed to valleys of height -2 . This transformation can be defined by induction on the length of the path. If $u$ has length 1 , then it consists of one horizontal step. In this case $T u$ is the path $(0,0) \rightarrow(1,-1)$. If $u$ has length 2 , the path $(0,0) \rightarrow(1,0) \rightarrow(2,0)$ is transformed into $(0,0) \rightarrow(1,-1) \rightarrow$ $(2,0)$, and the path $(0,0) \rightarrow(1,1) \rightarrow(2,0)$ remains fixed. Let now $v$ have length $n+1$ and let $T u$ be already defined for all paths of length $\leq n$. If $v$ is a path $u$ with a horizontal step added, there are two possibilities: If $T u$ ends in $(n, 0)$, then $T v$ is obtained by adding the step $(n, 0) \rightarrow(n+1,-1)$ to $T u$. If $T u$ ends in $(n,-1)$, then $T v$ is obtained by adding the step $(n,-1) \rightarrow(n+1,0)$ to $T u$. If on the other hand $v$ is a path $u$ augmented by $(n-1,0) \rightarrow(n, 1) \rightarrow(n+1,0)$, there are again two possibilities: If $T u$ ends in $(n-1,0)$, then $T v$ is obtained by adding the original peak $(n-1,0) \rightarrow$ $(n, 1) \rightarrow(n+1,0)$ to $T u$. If $T u$ ends in $(n-1,-1)$, then $T v$ is obtained by adding the valley (of height -2$)(n-1,-1) \rightarrow(n,-2) \rightarrow(n+1,-1)$ to $T u$. It is clear that $T$ is a bijection onto the set $A_{n}(2,-3)$ of all lattice paths in the strip $-3<y<2$ without horizontal steps, starting at $(0,0)$ and ending in $(n, 0)$ or $(n,-1)$. It is easily seen, that such a path has precisely $k=\left\lfloor\frac{n}{2}\right\rfloor$ northeast steps and $\ell=\left\lfloor\frac{n+1}{2}\right\rfloor$ southeast steps.

If $v$ is such a lattice path its induced weight is given by

$$
w_{t}(v)=q^{i_{1}+\cdots+i_{s}} t^{s}
$$

where the points with $x$-coordinates $i_{j}$ are either the peaks of height 1 or the valleys of height -2 of $v$. With this weight we have

$$
w_{t}\left(A_{n}(2,-3)\right)=F_{n+1}(q t) .
$$

Let now $A_{n}^{0}$ denote the set of all lattice paths with $k=\left\lfloor\frac{n}{2}\right\rfloor$ northeast steps and $\ell=\left\lfloor\frac{n+1}{2}\right\rfloor$ southeast steps. Let further $A_{n}^{m}(2,-3)$ (respectively $\left.A_{n}^{m}(-3,2)\right)$ be the set of all paths in $A_{n}^{0}$ where at least $m$ points are outside the strip such that the height of the first (from left to right) such point is $\geq 2$ (respectively $\leq-3$ ), the height of the second point is $\leq-3$ (respectively $\geq$ 2 ), the height of the next point is again $\geq 2$ (respectively $\leq-3$ ), and so on. Thus each path in $A_{n}^{m}(2,-3)$ and $A_{n}^{m}(-3,2)$ leaves the strip $-3<y<2$ at least $m$ times and oscillates between points above the strip and below the strip. By a simple inclusion-exclusion argument (cf. e.g. [7]) it is clear that 


$$
\begin{aligned}
& w_{t}\left(A_{n}(2,-3)\right)=w_{t}\left(A_{n}^{0}\right)+\sum_{m \geq 1}(-1)^{m} w_{t}\left(A_{n}^{m}(2,-3)\right)+ \\
& +\sum_{m \geq 1}(-1)^{m} w_{t}\left(A_{n}^{m}(-3,2)\right),
\end{aligned}
$$

if we extend the weight in a suitable manner to paths which are not contained in the strip. This will be done in (3.1). In the next paragraph we shall determine the weights of the sets $A_{n}^{m}$. Since the reflection principle (cf. [11]) does not work with these weights we adapt some ideas from [7] and [10] to this case.

\section{The main lemma}

Let $S(k, \ell)$ be the set of all lattice paths $v$ in $\mathbb{R}^{2}$ with $k$ northeast steps and $\ell$ southeast steps starting at $(0,0)$. The peaks with height at least 1 and the valleys with height at most -2 are called extremal points. Let $D(v)$ be the set of the $x$-coordinates of the extremal points of the path. Let $d(v)=|D(v)|$ and $\iota(v)=\sum_{i \in D(v)} i$. For a path $v \in S(k, \ell)$ we define the weight by

$$
w_{t}(v)=q^{\iota(v)} t^{d(v)} .
$$

For lattice paths in the strip $-3<y<2$ this coincides with the former definition.

A lattice path $v \in S(k, \ell)$ is uniquely determined by its extremal points.

It suffices to show that the path from an extremal point $(a, b)$ to the next extremal point $(x, y)$ is uniquely determined. Consider first the case that $(a, b)$ and $(x, y)$ are peaks. Then $(x-1, y-1)$ satisfies $y-1 \geq 0$. Let $k_{1}$ be the number of northeast steps and $\ell_{1}$ the number of southeast steps of the path up to the point $(x-1, y-1)$. Then we have $x-1-a=k_{1}+\ell_{1}$ and $y-b-1=k_{1}-\ell_{1}$. It suffices to show that there is precisely one path with $k_{1}$ northeast steps and $\ell_{1}$ southeast steps which contains no extremal point. In such a path each peak must have height $\leq 0$ and each valley height $\geq-1$. If $\ell_{1} \leq b$ this path begins with $\ell_{1}$ southeast steps followed by $k_{1}$ northeast steps. If $\ell_{1}>b$ the path must begin with $b$ southeast steps followed by $m=\ell_{1}-b$ zig-zag steps, i.e. pairs consisting of one southeast step and one northeast step. The path ends with $k_{1}-m=k_{1}-\ell_{1}+b=y-1 \geq 0$ northeast steps. If $(a, b)$ is a valley the path begins with $|b|+1$ northeast steps followed by $\ell_{1}$ zig-zag steps and the rest are northeast steps. The case that $(x, y)$ is a valley can be treated in an analogous way. 
A lattice path is thus uniquely determined by the sequence

$$
\left\{\left(k_{1}, l_{1}\right),\left(k_{2}, \ell_{2}\right), \cdots,\left(k_{s}, \ell_{s}\right)\right\},
$$

where $k_{i}$ (respectively $\ell_{i}$ ) is the number of northeast (respectively southeast) steps up to the point before the $i^{\prime}$ th extremal point. These numbers satisfy

$$
0 \leq k_{1}<k_{2}<\ldots<k_{s}<k, 0 \leq \ell_{1}<\ell_{2}<\ldots<\ell_{s}<\ell .
$$

On the other hand each pair of such sequences defines a unique lattice path in $S(k, \ell)$ with $s$ extremal points.

Let $u$ be the lattice path defined by $\left(k_{i}\right)$ and $\left(\ell_{i}\right)$. Its weight is

$$
w_{t}(u)=q^{s+\sum\left(k_{i}+\ell_{i}\right)} t^{s} .
$$

The total weight of the set of all lattice paths in $S(k, \ell)$ with $s$ extremal points is therefore

$$
q^{s^{2}}\left[\begin{array}{l}
k \\
s
\end{array}\right]\left[\begin{array}{l}
\ell \\
s
\end{array}\right] t^{s}
$$

This is an immediate consequence of the $q$-binomial theorem $(1+x)(1+q x) \cdots\left(1+q^{n-1} x\right)=\sum_{k \in \mathbb{Z}} q^{\left(\begin{array}{c}k \\ 2\end{array}\right)}\left[\begin{array}{l}n \\ k\end{array}\right] x^{k}$.

The left hand side may be written in the form $\sum_{s \geq 0} \sum_{0 \leq i_{1}<\cdots<i_{s}<n} q^{i_{1}+\cdots+i_{s}} x^{s}$. Comparing coefficients gives

$$
\sum_{0 \leq i_{1}<\cdots<i_{s}<n} q^{i_{1}+\cdots+i_{s}}=q^{\left(\begin{array}{c}
s \\
2
\end{array}\right)}\left[\begin{array}{c}
n \\
s
\end{array}\right] .
$$

Therefore we have

$$
w_{t}(S(k, \ell))=\sum q^{s^{2}}\left[\begin{array}{l}
k \\
s
\end{array}\right]\left[\begin{array}{l}
l \\
s
\end{array}\right] t^{s} .
$$

For $t=1$ the $q$-Vandermonde formula gives

$$
\begin{aligned}
& w_{1}(S(k, \ell))=\sum q^{s^{2}}\left[\begin{array}{l}
k \\
s
\end{array}\right]\left[\begin{array}{l}
\ell \\
s
\end{array}\right] \\
& =\sum q^{s^{2}}\left[\begin{array}{c}
k \\
k-s
\end{array}\right]\left[\begin{array}{l}
\ell \\
s
\end{array}\right]=\left[\begin{array}{c}
k+\ell \\
k
\end{array}\right] .
\end{aligned}
$$

Let $f_{s}\left(k, \ell, t, r_{1},-r_{2}, r_{3}, \cdots,(-1)^{n-1} r_{n}\right)$ be the weight of all lattice paths from $(0,0)$ to $(k+\ell, k-\ell)$ for which the sequence of extremal points has $s$ elements $P_{1}, \cdots, P_{s}$ and there exists a subsequence of $n$ elements $i_{1}<i_{2}<$ $\cdots<i_{n}$ such that the height $h\left(P_{i_{j}}\right)$ of $P_{i_{j}}$ satisfies $h\left(P_{i_{j}}\right) \geq r_{j} \geq 1$ if $j$ is 
odd and $h\left(P_{i_{j}}\right) \leq-r_{j} \leq-2$ for $j$ even, where $r_{j}$ are positive integers. In the same way $f_{s}\left(k, \ell, t,-r_{1}, r_{2}, r_{3}, \cdots,(-1)^{n} r_{n}\right)$ denotes the weight of those lattice paths where $h\left(P_{i_{j}}\right) \geq r_{j} \geq 1$ if $j$ is even and $h\left(P_{i_{j}}\right) \leq-r_{j} \leq-2$ for $j$ odd.

Let $x_{n}:=r_{1}+\cdots+r_{n}, y_{n}: r_{n}+3 r_{n-1}+5 r_{n-2}+\cdots$, such that $x_{n}=x_{n-1}+r_{n}$ and $y_{n}=y_{n-1}+2 x_{n-1}+r_{n}$.

We show the following

\section{Lemma 3.1}

For $k-\ell<r_{n}$ we have

$$
\begin{aligned}
& f\left(k, \ell, t, r_{1}, \cdots,-r_{n-1}, r_{n}\right) \\
& =q^{y_{n}} \sum_{s \geq n} q^{(s-n)(s+n)}\left[\begin{array}{c}
k-x_{n}+2 n \\
s+n
\end{array}\right]\left[\begin{array}{c}
\ell+x_{n}-2 n \\
s-n
\end{array}\right] t^{s}
\end{aligned}
$$

and

$$
\begin{aligned}
& f\left(k, \ell, t,-r_{1}, \cdots,-r_{n-1}, r_{n}\right) \\
& =q^{y_{n}} \sum_{s \geq n} q^{(s-n)(s+n)}\left[\begin{array}{c}
k-x_{n}+2 n \\
s+n
\end{array}\right]\left[\begin{array}{c}
\ell+x_{n}-2 n \\
s-n
\end{array}\right] t^{s} .
\end{aligned}
$$

For $k-\ell>-r_{n}$ we have

$$
\begin{aligned}
& f\left(k, \ell, t, r_{1}, \cdots, r_{n-1},-r_{n}\right) \\
& =q^{y_{n}} \sum_{s \geq n} q^{(s-n)(s+n)}\left[\begin{array}{c}
\ell-x_{n}+2 n \\
s+n
\end{array}\right]\left[\begin{array}{c}
k+x_{n}-2 n \\
s-n
\end{array}\right] t^{s}
\end{aligned}
$$

and

$$
\begin{aligned}
& f\left(k, \ell, t,-r_{1}, \cdots, r_{n-1},-r_{n}\right) \\
& =q^{y_{n}} \sum_{s \geq n} q^{(s-n)(s+n)}\left[\begin{array}{c}
\ell-x_{n}+2 n \\
s+n
\end{array}\right]\left[\begin{array}{c}
k+x_{n}-2 n \\
s-n
\end{array}\right] t^{s} .
\end{aligned}
$$

Proof Let $I_{0}=0, I_{1}$ be the smallest index $i$ such that $k_{i}-\ell_{i}>r_{1}-2, I_{2}$ the smallest $i>I_{1}$ such that $k_{i}-\ell_{i}<-r_{2}+2, I_{3}$ the smallest $i>I_{2}$ such that $k_{i}-\ell_{i}>r_{3}-2$, and so on. Moreover let $I_{n+1}=s$. This defines a splitting of $\left(k_{i}\right)_{i=1}^{S}$ and $\left(\ell_{i}\right)_{i=1}^{S}$ into $n+1$ segments:

$$
k_{1}, \cdots, k_{I_{1}-1}, k_{I_{1}},\left|k_{I_{1}+1}, \cdots, k_{I_{2}}, \cdots\right| k_{I_{n}+1}, \cdots, k_{s}
$$

and

$$
\ell_{1}, \cdots, \ell_{I_{1}-1}, \ell_{I_{1}},\left|\ell_{I_{1}+1}, \cdots, \ell_{I_{2}},\right| \cdots \mid \ell_{I_{n}+1}, \cdots, \ell_{s} .
$$

Let $K_{j}=\left(k_{I_{j-1}+1}, \cdots, k_{I_{j}}\right)$ be the $j^{\prime}$ th segment $(j \geq 1)$ of the first sequence and $L_{j}=\left(\ell_{I_{j-1}+1}, \cdots, \ell_{I_{j}}\right)$ the $j^{\prime}$ th segment of the second sequence. For 
odd $j$ we have $k_{i}-\ell_{i} \leq r_{j}-2$ for $I_{j-1} \leq i<I_{j}$ and $k_{I_{j}}-\ell_{I_{j}}>r_{j}-2$. If $j$ is even we have $k_{i}-\ell_{i} \geq-r_{j}+2$ for $I_{j-1} \leq i<I_{j}$ and $k_{I_{j}}-\ell_{I_{j}}<-r_{j}+2$. Now we associate with $\left(k_{i}\right)_{i=1}^{s}$ and $\left(\ell_{i}\right)_{i=1}^{s}$ two new sequences $\left(k_{i}^{n}\right)_{i=1}^{s-n}$ and $\left(\ell_{i}^{n}\right)_{i=1}^{s+n}$ in the following way: Let $\left(k_{i}^{n}\right)_{i=1}^{s-n}$ be the sequence

$$
\begin{aligned}
& \left(k_{i}^{n}\right)_{i=1}^{s-n}=k_{1}, \cdots, k_{I_{1}-1}, \mid \ell_{I_{1}+1}+x_{1}-2, \cdots, \\
& \ell_{I_{2}-1}+x_{1}-2\left|k_{I_{2}+1}+x_{2}-4, \cdots, k_{I_{3}-1}+x_{2}-4,\right| \cdots .
\end{aligned}
$$

Let $K_{j}^{n}$ be the $j^{\prime}$ th segment of this sequence. For odd $j$ it is given by $k_{I_{j-1}+1}+x_{j-1}-2(j-1), \cdots, k_{I_{j-1}}+x_{j-1}-2(j-1)$ and for even $j$ by $\ell_{I_{j-1}+1}+x_{j-1}-2(j-1), \cdots, \ell_{I_{j-1}}+x_{j-1}-2(j-1)$. If $I_{j+1}=I_{j}+1$ then the corresponding segment is empty.

The sequence $\left(k_{i}^{n}\right)_{i=1}^{s-n}$ is again strictly increasing. We have to show that the last element of each segment is less than the first element of the next segment which is not empty. If $j$ is even it suffices to show that $\ell_{I_{j}-1}+$ $x_{j-1}-2(j-1)<k_{I_{j}+1}+x_{j}-2 j$. This follows from $k_{I_{j}-1}-\ell_{I_{j}-1} \geq$ $-r_{j}+2=x_{j-1}-2(j-1)-x_{j}+2 j$ and $k_{I_{j}+1}>k_{I_{j}-1}$. For odd $j$ we have to show that $k_{I_{j}-1}+x_{j-1}-2(j-1)<l_{I_{j}+1}+x_{j}-2 j$. This follows from $k_{I_{j}-1}-\ell_{I_{j}-1} \leq r_{j}-2=-x_{j-1}+2(j-1)+x_{j}-2 j$ and $\ell_{I_{j}-1}<\ell_{I_{j}+1}$.

In an analogous way $\left(\ell_{i}^{n}\right)_{i=1}^{s+n}$ is defined by

$$
\begin{aligned}
& \left(\ell_{i}^{n}\right)_{i=1}^{s+n}=\ell_{1}, \cdots, \ell_{I_{1}}, \mid k_{I_{1}}-x_{1}+2, \cdots, k_{I_{2}}-x_{1}+2, \\
& \left|\ell_{I_{2}}-x_{2}+4, \cdots, \ell_{I_{3}}-x_{2}+4,\right| \cdots .
\end{aligned}
$$

Let $L_{j}^{n}$ denote the $j^{\prime}$ th segment of this sequence. For even $j$ it is given by $k_{I_{j-1}}-x_{j-1}+2(j-1), \cdots, k_{I_{j}}-x_{j-1}+2(j-1)$ and for odd $j$ by $\ell_{I_{j-1}}-x_{j-1}+2(j-1), \cdots, \ell_{I_{j}}-x_{j-1}+2(j-1)$.

The sequence $\left(\ell_{i}^{n}\right)_{i=1}^{s+n}$ is strictly increasing too. Here we have to show that for even $j$ we have $k_{I_{j}}-x_{j-1}+2(j-1)<\ell_{I_{j}}-x_{j}+2 j$ or $k_{I_{j}}-\ell_{I_{j}}<-r_{j}+2$ which holds by definition. For odd $j$ we must show that $\ell_{I_{j}}-x_{j-1}+2(j-1)<$ $k_{I_{j}}-x_{j}+2 j$ which is also satisfied.

From the sequences $\left(k_{i}^{n}\right)_{i=1}^{s-n}$ and $\left(\ell_{i}^{n}\right)_{i=1}^{s+n}$ the original sequences can be reconstructed. It suffices to show that the values $I_{1}, I_{2}, \cdots$ can be determined. To this end we add $2 n$ elements $\infty$, the latter denoting an element greater than any integer, at the end of $\left(k_{i}^{n}\right)_{i=1}^{s-n}$. Then consider the difference of the first element of $K_{j+1}^{n}$ and the last element of $L_{j}^{n}$. For even $j$ this is $k_{I_{j}+1}+x_{j}-2 j-\left(k_{I_{j}}-x_{j-1}+2(j-1)\right)>x_{j}+x_{j-1}-4 j+2$, and for odd $j$ we have $\ell_{I_{j}+1}+x_{j}-2 j-\left(\ell_{I_{j}}-x_{j-1}+2(j-1)\right)>x_{j}+x_{j-1}-4 j+2$. (If $K_{j+1}^{n}$ is empty we choose instead the first element of the next segment). Consider first the sequence $\left(k_{i}^{n}-\ell_{i}^{n}\right)$. Here $I_{1}$ is the smallest number $i$ such that $k_{i}^{n}-\ell_{i}^{n}>x_{1}-2=r_{1}-2$. Therefore we know $\ell_{I_{1}}=\ell_{I_{1}}^{n}$ and $k_{I_{1}}=\ell_{I_{1}+1}^{n}+x_{1}-2$ and therefore also the first segments of the original sequences. Then we cancel all elements from $\left(k_{i}^{n}\right)_{i=1}^{s-n}$ and $\left(\ell_{i}^{n}\right)_{i=1}^{s+n}$ containing 
some $k_{i}, \ell_{i}$ with $i \leq I_{1}$, i.e. we consider the sequences

$\left|\ell_{I_{1}+1}+x_{1}-2, \cdots, \ell_{I_{2}-1}+x_{1}-2,\right| k_{I_{2}+1}+x_{2}-4, \cdots, k_{I_{3}-1}+x_{2}-4, \mid \cdots$ and

$\left|k_{I_{1}+1}-x_{1}+2, \cdots, k_{I_{2}}-x_{1}+2,\right| \ell_{I_{2}}-x_{2}+4, \cdots, l_{I_{3}}-x_{2}+4, \mid \cdots$.

For $i<I_{2}$ we have

$\left(\ell_{i}+x_{1}-2\right)-\left(k_{i}-x_{1}+2\right)=\left(\ell_{i}-k_{i}\right)+2 x_{1}-4<\left(r_{2}+x_{1}\right)+x_{1}-4-2$.

Therefore $I_{2}$ is the smallest $i$ of this sequence, where the difference is $>$ $x_{2}+x_{1}-4-2$. This gives the elements $k_{I_{2}}$ and $\ell_{I_{2}}$ and therefore the second segment of the original sequence, and so on.

The same procedure can be applied for arbitrary strictly increasing sequences $\left(k_{i}^{n}\right)_{i=1}^{s-n}$ and $\left(\ell_{i}^{n}\right)_{i=1}^{s+n}$. It results in two sequences $\left(k_{i}\right)_{i=1}^{s}$ and $\left(\ell_{i}\right)_{i=1}^{s}$. It remains to show that these sequences are again strictly increasing. As above we determine $I_{1}$ and define $\ell_{i}=\ell_{i}^{n}, 1 \leq i \leq I_{1}$, and $k_{i}=k_{i}^{n}$ for $i<I_{1}$ and $k_{I_{1}}=\ell_{I_{1}+1}^{n}+x_{1}-2$. We have to show that $k_{I_{1}-1}<k_{I_{1}}=\ell_{I_{1}+1}^{n}+x_{1}-2$. But this follows from $k_{I_{1}-1}^{n}-\ell_{I_{1}-1}^{n}<r_{1}-2$ and so on.

Thus we get a bijection from the set of all pairs of strictly increasing sequences $\left(k_{i}\right)_{i=1}^{s}$ with $k_{s}<k$ and $\left(\ell_{i}\right)_{i=1}^{s}$ with $\ell_{s}<\ell$ and all pairs of strictly increasing sequences $\left(k_{i}^{n}\right)_{i=1}^{s-n}$ with $k_{s-n}^{n}<K_{n}$ and $\left(\ell_{i}^{n}\right)_{i=1}^{s+n}$ with $\ell_{s+n}^{n}<L_{n}$. Here we have $K_{n}=k+x_{n}-2 n$ for even $n$ and $K_{n}=\ell+x_{n}-2 n$ for odd $n$ and $L_{n}=k-x_{n}+2 n$ for odd $n$ and $L_{n}=\ell-x_{n}+2 n$ for even $n$.

By comparing these sequences we see that

$$
\begin{aligned}
& \sum_{i} k_{i}+\sum_{j} \ell_{j}=2 \sum_{k=1}^{n-1}\left(x_{k}-2 k\right)+x_{n}-2 n+\sum_{i} k_{i}^{n}+\sum_{j} \ell_{j}^{n} \\
& =y_{n}-2 n^{2}+\sum_{i} k_{i}^{n}+\sum_{j} \ell_{j}^{n} .
\end{aligned}
$$

If $n$ is even we have $\sum q^{\sum_{i} k_{i}^{n}}=q^{\left(\begin{array}{c}s-n \\ 2\end{array}\right)}\left[\begin{array}{c}k+x_{n}-2 n \\ s-n\end{array}\right]$ and $\sum q^{\sum_{i} \ell_{i}^{n}}=q^{\left(\begin{array}{c}s+n \\ 2\end{array}\right)}\left[\begin{array}{c}\ell-x_{n}+2 n \\ s+n\end{array}\right]$.

This implies

$$
\begin{aligned}
& f_{s}\left(k, \ell, t, r_{1},-r_{2}, r_{3}, \cdots,-r_{n}\right)= \\
& =q^{s+y_{n}+\left(\begin{array}{c}
s+n \\
2
\end{array}\right)+\left(\begin{array}{c}
s-n \\
2
\end{array}\right)-2 n^{2}}\left[\begin{array}{c}
k+x_{n}-2 n \\
s-n
\end{array}\right]\left[\begin{array}{c}
\ell-x_{n}+2 n \\
s+n
\end{array}\right] t^{s}= \\
& =q^{y_{n}+(s-n)(s+n)}\left[\begin{array}{c}
k+x_{n}-2 n \\
s-n
\end{array}\right]\left[\begin{array}{c}
\ell-x_{n}+2 n \\
s+n
\end{array}\right] t^{s} .
\end{aligned}
$$

If $n$ is odd $k$ and $\ell$ change their roles. If the first height is $-r_{1}$ exchange the sequences $\left(k_{i}\right)$ and $\left(\ell_{i}\right)$. If $K_{n} \leq 0$ or $L_{n} \leq 0$ then there are no such lattice 
paths and the corresponding formula (3.18) also reduces to 0 , because of our general assumption about $q$-binomial coefficients.

In order to clarify the situation let us give a simple example. Let $k=3, \ell=$ $2, s=2$. There are $\left(\begin{array}{l}3 \\ 2\end{array}\right)\left(\begin{array}{l}2 \\ 2\end{array}\right)=3$ lattice paths in $S(3,2)$ with 2 extremal points. They correspond to the following pairs of increasing sequences:

a) $\left(k_{i}\right)=(0,1),\left(\ell_{i}\right)=(0,1)$,

b) $\left(k_{i}\right)=(0,2),\left(\ell_{i}\right)=(0,1)$,

c) $\left(k_{i}\right)=(1,2),\left(\ell_{i}\right)=(0,1)$.

The weight of this set ist $\left(q^{2+2}+q^{2+3}+q^{2+4}\right) t^{2}=q^{4}\left[\begin{array}{l}3 \\ 2\end{array}\right]\left[\begin{array}{l}2 \\ 2\end{array}\right] t^{2}$. Choose now $n=1$ and $r_{1}=2$ and a corresponding $i$ with $k_{i}-\ell_{i} \geq 1$. For the path a) there is no such point, for b) there is one such point and for c) both points satisfy this inequality.

The splitting into segments and the corresponding sequences $\left(k_{i}^{1}\right)$ and $\left(\ell_{i}^{1}\right)$ are

$\left(k_{i}\right)=02\left|\varnothing,\left(\ell_{i}\right)=01\right| \varnothing$

$\left(k_{i}^{1}\right)_{i=1}^{1}=(0),\left(\ell_{i}^{1}\right)_{i=1}^{3}=(0,1,2)$,

and

$\left(k_{i}\right)=1\left|2,\left(\ell_{i}\right)=0\right| 1$

$\left(k_{i}^{1}\right)_{i=1}^{1}=(1),\left(\ell_{i}^{1}\right)_{i=1}^{3}=(0,1,2)$.

The weight of this set is

$f_{2}(3,2, t, 2)=\left(q^{2+3}+q^{2+4}\right) t^{2}=q^{y_{1}} q^{(2-1)(2+1)}\left[\begin{array}{c}3-2+2 \\ 2+1\end{array}\right]\left[\begin{array}{c}2+2-2 \\ 2-1\end{array}\right] t^{2}$.

Now consider the lattice paths in $S(3,2)$ with $s=n=1, r_{1}=2$.

They correspond to the following pairs of sequences:

a) $\left(k_{i}\right)=(1),\left(\ell_{i}\right)=(0)$

b) $\left(k_{i}\right)=(2),\left(\ell_{i}\right)=(0)$

c) $\left(k_{i}\right)=(2),\left(\ell_{i}\right)=(1)$

The corresponding sequences $\left(k_{i}^{1}\right)$ and $\left(\ell_{i}^{1}\right)$ are

ad a) $\left(k_{i}^{1}\right)_{i=1}^{0}=\varnothing,\left(\ell_{i}^{1}\right)_{i=1}^{2}=(0,1)$,

ad b) $\left(k_{i}^{1}\right)_{i=1}^{0}=\varnothing,\left(\ell_{i}^{1}\right)_{i=1}^{2}=(0,2)$,

ad c) $\left(k_{i}^{1}\right)_{i=1}^{0}=\varnothing,\left(\ell_{i}^{1}\right)_{i=1}^{2}=(1,2)$. 


\section{Corollary 3.1}

For $k-\ell<r_{n}$ we get

$$
\begin{aligned}
& f\left(k, \ell, 1, r_{1}, \cdots,-r_{n-1}, r_{n}\right)=q^{y_{n}}\left[\begin{array}{c}
k+\ell \\
k-x_{n}
\end{array}\right]=q^{y_{n}}\left[\begin{array}{c}
k+\ell \\
\ell+x_{n}
\end{array}\right], \\
& f\left(k, \ell, 1,-r_{1}, \cdots,-r_{n-1}, r_{n}\right)=q^{y_{n}}\left[\begin{array}{c}
k+\ell \\
k-x_{n}
\end{array}\right]=q^{y_{n}}\left[\begin{array}{c}
k+\ell \\
\ell+x_{n}
\end{array}\right]
\end{aligned}
$$

and for $k-\ell>-r_{n}$

$$
\begin{gathered}
f\left(k, \ell, 1, r_{1}, \cdots, r_{n-1},-r_{n}\right)=q^{y_{n}}\left[\begin{array}{c}
k+\ell \\
k+x_{n}
\end{array}\right]=q^{y_{n}}\left[\begin{array}{c}
k+\ell \\
\ell-x_{n}
\end{array}\right], \\
f\left(k, \ell, 1,-r_{1}, \cdots, r_{n-1},-r_{n}\right)=q^{y_{n}}\left[\begin{array}{c}
k+\ell \\
k+x_{n}
\end{array}\right]=q^{y_{n}}\left[\begin{array}{c}
k+\ell \\
\ell-x_{n}
\end{array}\right] .
\end{gathered}
$$

This is a consequence of the $q$-Vandermonde formula, which gives

$$
\begin{gathered}
\sum_{s \geq n} q^{(s-n)(s+n)}\left[\begin{array}{c}
k-a+2 n \\
s+n
\end{array}\right]\left[\begin{array}{c}
\ell+a-2 n \\
s-n
\end{array}\right]= \\
\sum_{s \geq n} q^{(s-n)(s+n)}\left[\begin{array}{c}
k-a+2 n \\
k-a+n-s
\end{array}\right]\left[\begin{array}{c}
\ell+a-2 n \\
s-n
\end{array}\right]=\left[\begin{array}{c}
k+\ell \\
k-a
\end{array}\right] .
\end{gathered}
$$

If we choose $a=0$ and let $k \rightarrow \infty, \ell \rightarrow \infty$ we get the well-known formula

$$
\sum_{s \geq n} q^{(s-n)(s+n)} \frac{1}{(q)_{s-n}(q)_{s+n}}=\frac{1}{(q)_{\infty}}
$$

if we set as usual $(q)_{n}=(1-q) \cdots\left(1-q^{n}\right)$ and $(q)_{\infty}=(1-q)\left(1-q^{2}\right) \cdots$. (In order to make sense of the limit we can either choose $|q|<1$ or we regard $q$ as an indeterminate and interpret both sides as formal power series. In the latter case convergence of $\sum a_{n, k} q^{k} \rightarrow \sum a_{k} q^{k}$ means that for each $k$ there exists $N(k)$ such that $a_{n, k}=a_{k}$ for all $\left.n \geq N(k)\right)$.

Such limit relations give us some interesting formulas. Let $S(k,-)$ be the set of all lattice paths with $k$ northeast steps and an arbitrary finite number of southeast steps then from (3.8) we get

$w_{1}(S(k,-))=\frac{1}{(q)_{k}}$

and from (3.7)

$w_{t}(S(k,-))=\sum_{s \geq 0}\left[\begin{array}{l}k \\ s\end{array}\right] \frac{q^{s^{2}}}{(q)_{s}} t^{s}$.

For the set $S(-,-)$ of all lattice paths with an arbitrary finite number of 
northeast and southeast steps we get

$w_{1}(S(-,-))=\frac{1}{(q)_{\infty}}$ and $w_{t}(S(-,-))=\sum_{s \geq 0} \frac{q^{s^{2}}}{(q)_{s}^{2}} t^{s}$.

From Lemma 3.1 we deduce in the same way $f\left(-,-, t, r_{1}, \cdots, \pm r_{n}\right)=q^{y_{n}} \sum_{s \geq n} q^{(s-n)(s+n)} \frac{t^{s}}{(q)_{s-n}(q)_{s+n}}$ and $f\left(-,-, 1, r_{1}, \cdots, \pm r_{n}\right)=\frac{q^{y_{n}}}{(q)_{\infty}}$.

\section{The Rogers-Ramanujan identities}

Let now $k=\left\lfloor\frac{n}{2}\right\rfloor$ and $\ell=\left\lfloor\frac{n+1}{2}\right\rfloor$ and choose $r_{2 k+1}=2, r_{2 k}=3$. Then we have $x_{2 i}=5 i, x_{2 i+1}=5 i+2, y_{i}=\frac{i(5 i-1)}{2}$. In order to avoid misunderstandings we mention again that we assume that the $q$-binomial coefficients $\left[\begin{array}{l}n \\ k\end{array}\right]=0$ if $n<0$. Therefore we get

$$
\begin{aligned}
& w_{t}\left(A_{n}^{2 i+1}(2,-3)\right)=f\left(k, \ell, t, r_{1}, \cdots, r_{2 i+1}\right)= \\
& =q^{y_{2 i+1}} \sum_{s \geq 2 i+1} q^{(s+2 i+1)(s-2 i-1)} \\
& {\left[\begin{array}{c}
\left\lfloor\frac{n}{2}\right\rfloor-x_{2 i+1}+2(2 i+1) \\
s+2 i+1
\end{array}\right]\left[\begin{array}{c}
\left\lfloor\frac{n+1}{2}\right\rfloor+x_{2 i+1}-2(2 i+1) \\
s-2 i-1
\end{array}\right] t^{s}=} \\
& =q^{y_{2 i+1}} \sum_{s \geq 2 i+1} q^{(s+2 i+1)(s-2 i-1)}\left[\begin{array}{c}
\left\lfloor\frac{n}{2}\right\rfloor-i \\
s+2 i+1
\end{array}\right]\left[\begin{array}{c}
\left\lfloor\frac{n+1}{2}\right\rfloor+i \\
s-2 i-1
\end{array}\right] t^{s}= \\
& =q^{y_{2 i+1}} \sum_{s \geq 2 i+1} q^{(s+2 i+1)(s-2 i-1)}\left[\begin{array}{c}
\left\lfloor\frac{n-(2 i+1)+1}{2}\right\rfloor \\
s+2 i+1
\end{array}\right]\left[\begin{array}{c}
\left\lfloor\frac{n+2 i+1}{2}\right\rfloor \\
s-2 i-1
\end{array}\right] t^{s} .
\end{aligned}
$$

In the same way we get

$$
\begin{aligned}
& w_{t}\left(A_{n}^{2 i}(2,-3)\right)=f\left(k, \ell, t, r_{1},-r_{2}, \cdots,-r_{2 i}\right)= \\
& q^{y_{2 i}} \sum_{s \geq 2 i} q^{(s-2 i)(s+2 i)}\left[\begin{array}{c}
\left\lfloor\frac{n+2 i}{2}\right\rfloor \\
s-2 i
\end{array}\right]\left[\begin{array}{c}
\left\lfloor\frac{n-2 i+1}{2}\right\rfloor \\
s+2 i
\end{array}\right] t^{s} .
\end{aligned}
$$

Therefore in either case we have

$$
\begin{aligned}
& w_{t}\left(A_{n}^{i}(2,-3)\right)=f\left(k, \ell, t, r_{1}, \cdots,(-1)^{i-1} r_{i}\right)= \\
& q^{\frac{i(5 i-1)}{2}} \sum_{s \geq i} q^{(s-i)(s+i)}\left[\begin{array}{c}
\left\lfloor\frac{n+i}{2}\right\rfloor \\
s-i
\end{array}\right]\left[\begin{array}{c}
\left\lfloor\frac{n-i+1}{2}\right\rfloor \\
s+i
\end{array}\right] t^{s} .
\end{aligned}
$$

Analogously we find 


$$
\begin{aligned}
& w_{t}\left(A_{n}^{i}(-3,2)\right)=f\left(k, \ell, t,-r_{2}, \cdots,(-1)^{i} r_{i+1}\right)= \\
& q^{\frac{i(5 i+1)}{2}} \sum_{s \geq i} q^{(s-i)(s+i)}\left[\begin{array}{c}
\left\lfloor\frac{n-i}{2}\right\rfloor \\
s+i
\end{array}\right]\left[\begin{array}{c}
\left\lfloor\frac{n+i+1}{2}\right\rfloor \\
s-i
\end{array}\right] t^{s} .
\end{aligned}
$$

If we set

$$
f_{i}(n, t)=q^{\frac{i(5 i-1)}{2}} \sum_{s \geq i} q^{(s-i)(s+i)}\left[\begin{array}{c}
\left\lfloor\frac{n+i}{2}\right\rfloor \\
s-i
\end{array}\right]\left[\begin{array}{c}
\left\lfloor\frac{n-i+1}{2}\right\rfloor \\
s+i
\end{array}\right] t^{s}
$$

for $i \in \mathbb{Z}$, then for $i>0$

$w_{t}\left(A_{n}^{i}(2,-3)\right)=f_{i}(n, t)$

and

$w_{t}\left(A_{n}^{i}(-3,2)\right)=f_{-i}(n, t)$.

Therefore we get from (2.12)

\section{Theorem 4.1}

Let

$f_{i}(n, t)=q^{\frac{i(5 i-1)}{2}} \sum_{s \geq|i|} q^{(s-i)(s+i)}\left[\begin{array}{c}\left\lfloor\frac{n+i}{2}\right\rfloor \\ s-i\end{array}\right]\left[\begin{array}{c}\left\lfloor\frac{n-i+1}{2}\right\rfloor \\ s+i\end{array}\right] t^{s}$.

Then we have

$$
\begin{aligned}
& F_{n+1}(q t)=w_{t}\left(A_{n}(2,-3)\right)= \\
& \sum_{i \in \mathbb{Z}}(-1)^{i} f_{i}(n, t)=\sum_{i=-n}^{n+1}(-1)^{i} f_{i}(n, t) .
\end{aligned}
$$

Comparing coefficients we get the identity

$$
\begin{aligned}
& \sum_{i \in \mathbb{Z}}(-1)^{i} q^{\frac{i(3 i-1)}{2}}\left[\begin{array}{c}
\left\lfloor\frac{n+i}{2}\right\rfloor \\
s-i
\end{array}\right]\left[\begin{array}{c}
\left\lfloor\frac{n-i+1}{2}\right\rfloor \\
s+i
\end{array}\right]= \\
& \sum_{i=-n}^{n+1}(-1)^{i} q^{\frac{i(3 i-1)}{2}}\left[\begin{array}{c}
\left\lfloor\frac{n+i}{2}\right\rfloor \\
s-i
\end{array}\right]\left[\begin{array}{c}
\left\lfloor\frac{n-i+1}{2}\right\rfloor \\
s+i
\end{array}\right]=\left[\begin{array}{c}
n-s \\
s
\end{array}\right] .
\end{aligned}
$$

The identity (4.3) reduces for $n \rightarrow \infty$ to

$$
\sum_{i=-s}^{s}(-1)^{i} q^{\frac{i(3 i-1)}{2}}\left[\begin{array}{c}
2 s \\
s-i
\end{array}\right]=\left(1-q^{s+1}\right) \cdots\left(1-q^{2 s}\right) .
$$


This is a well-known finite version of Euler's pentagonal number theorem

$$
\frac{1}{(q)_{\infty}} \sum_{i \in \mathbb{Z}}(-1)^{i} q^{\frac{i(3 i-1)}{2}}=1
$$

which is obtained by letting $s \rightarrow \infty$.

Corollary 4.1 (Finite version of the first Rogers-Ramanujan identity, see [18], [2])

$$
\sum_{k=0}^{\left\lfloor\frac{n}{2}\right\rfloor} q^{k^{2}}\left[\begin{array}{c}
n-k \\
k
\end{array}\right]=F_{n+1}(q)=\sum_{i \in \mathbb{Z}}(-1)^{i} q^{\frac{i(5 i-1)}{2}}\left[\begin{array}{c}
n \\
\left\lfloor\frac{n+5 i}{2}\right\rfloor
\end{array}\right] .
$$

This result follows immediately by setting $t=1$ in (4.2) and using the $q$-Vandermonde formula.

If we let $n \rightarrow \infty$ we get the first Rogers-Ramanujan identity

$$
\sum_{k \geq 0} \frac{q^{k^{2}}}{(1-q) \cdots\left(1-q^{k}\right)}=\frac{1}{(q)_{\infty}} \sum_{i \in \mathbb{Z}}(-1)^{i} q^{\frac{i(5 i-1)}{2}} .
$$

In order to obtain the second Rogers-Ramanujan identity we consider the set $B_{n}(-3,2)$ of all lattice paths without horizontal steps which start in $(0,1)$, remain in the strip $-2 \leq y \leq 1$ and end in $(n,-1)$ or $(n, 0)$. Their weight is of course $F_{n}\left(q^{2} t\right)$. Now we shift each such lattice path one unit downward, such that the new path starts in $(0,0)$. In order that the new path has the same weight as the old path we have to exchange in the new path the peaks of height 0 and the valleys of height -2 . For under this map all extremal points are again mapped onto extremal points, except the peaks of height 1 which are mapped onto peaks of height 0 . If we replace the latter peaks with the corresponding valleys of height -2 , we get again an extremal point. On the other hand the image of a valley of height -1 , which is not an extremal point, is mapped onto a valley of height -2 , which is an extremal point. So we replace it with the corresponding peak of height 0 , such that the weights are preserved. In this way we obtain the set $A_{n}(-4,1)$ of all lattice paths starting at $(0,0)$ which remain in the strip $-3 \leq y \leq 0$.

In this case we can apply the reasoning above with $r_{2 i+1}=1, r_{2 i}=4$. This gives $x_{2 i}=5 i, x_{2 i+1}=5 i+1$ and $y_{i}=\frac{(5 i-3) i}{2}$. There are $k=\left\lfloor\frac{n-1}{2}\right\rfloor$ northeast steps and $l=\left\lfloor\frac{n+2}{2}\right\rfloor$ southeast steps.

We therefore get 
Theorem 4.2 Let

$$
g_{i}(n, t)=q^{3}\left(\begin{array}{c}
i \\
2
\end{array}\right) \sum_{s \geq|i|} q^{s^{2}}\left[\begin{array}{c}
\left\lfloor\frac{n+i-1}{2}\right\rfloor \\
s-i
\end{array}\right]\left[\begin{array}{c}
\left\lfloor\frac{n-i+2}{2}\right\rfloor \\
s+i
\end{array}\right] t^{s} .
$$

Then we have

$$
F_{n}\left(q^{2} t\right)=\sum_{i \in \mathbb{Z}}(-1)^{i} g_{i}(n, t)=\sum_{i=-n+1}^{n+2}(-1)^{i} g_{i}(n, t) .
$$

Comparing coefficients we get

$$
\begin{aligned}
& \sum_{i \in \mathbb{Z}}(-1)^{i} q^{3\left(\begin{array}{l}
i \\
2
\end{array}\right)}\left[\begin{array}{c}
\left\lfloor\frac{n+i}{2}\right\rfloor \\
s-i
\end{array}\right]\left[\begin{array}{c}
\left\lfloor\frac{n-i+3}{2}\right\rfloor \\
s+i
\end{array}\right]= \\
& \sum_{i=-n+1}^{n+2}(-1)^{i} q^{3}\left(\begin{array}{c}
i \\
2
\end{array}\right)\left[\begin{array}{c}
\left\lfloor\frac{n+i}{2}\right\rfloor \\
s-i
\end{array}\right]\left[\begin{array}{c}
\left\lfloor\frac{n-i+3}{2}\right\rfloor \\
s+i
\end{array}\right]=q^{s}\left[\begin{array}{c}
n-s \\
s
\end{array}\right] .
\end{aligned}
$$

For $n \rightarrow \infty$ this reduces to the identity

$$
\sum_{i=-s}^{s}(-1)^{i} q^{3\left(\begin{array}{c}
i \\
2
\end{array}\right)}\left[\begin{array}{c}
2 s \\
s-i
\end{array}\right]=q^{s}\left(1-q^{s+1}\right) \cdots\left(1-q^{2 s}\right) .
$$

Corollary 4.2 (Finite version of the second identity of RogersRamanujan, see [18], [2])

$$
\begin{aligned}
& \sum_{k=0}^{\left\lfloor\frac{n-1}{2}\right\rfloor} q^{k^{2}+k}\left[\begin{array}{c}
n-k-1 \\
k
\end{array}\right]=F_{n}\left(q^{2}\right)=\sum_{i \in \mathbb{Z}}(-1)^{i} q^{\frac{i(5 i-3)}{2}} \\
& {\left[\begin{array}{c}
n \\
\left\lfloor\frac{n-5 i+2}{2}\right\rfloor
\end{array}\right] .}
\end{aligned}
$$

By letting $n \rightarrow \infty$ we get the second Rogers-Ramanujan identity

$$
\sum_{k \geq 0} \frac{q^{k^{2}+k}}{(1-q) \cdots\left(1-q^{k}\right)}=\frac{1}{(q)_{\infty}} \sum_{i \in \mathbb{Z}}(-1)^{i} q^{\frac{i(5 i-3)}{2}} .
$$

With the same method we get a slight generalization of another result by I. Schur [18:

Let $k=\left\lfloor\frac{n}{2}\right\rfloor, \ell=\left\lfloor\frac{n+1}{2}\right\rfloor$ and $r_{2 k+1}=1, r_{2 k}=2$. Then there is only one lattice path starting at $(0,0)$ and remaining in the strip $-1 \leq y \leq 0$. It has weight 1 . In this case we have $x_{2 i}=3 i, x_{2 i+1}=3 i+1, y_{i}=\frac{i(3 i-1)}{2}$.

Here we get

$$
\begin{aligned}
& f\left(k, \ell, t, r_{1},-r_{2}, \cdots,(-1)^{i-1} r_{i}\right)=q^{\frac{i(3 i-1)}{2}} \sum_{s \geq i} q^{(s-i)(s+i)} \\
& {\left[\begin{array}{c}
\left\lfloor\frac{n+i+1}{2}\right\rfloor \\
s+i
\end{array}\right]\left[\begin{array}{c}
\left\lfloor\frac{n-i}{2}\right\rfloor \\
s-i
\end{array}\right] t^{s} .}
\end{aligned}
$$


This gives

\section{Theorem 4.3}

For each $n$ we have

$$
\sum_{i \in \mathbb{Z}}(-1)^{i} q^{\frac{i(3 i-1)}{2}}\left[\begin{array}{c}
n \\
\left\lfloor\frac{n+3 i}{2}\right\rfloor
\end{array}\right]=1 .
$$

Setting

$$
h_{i}(n, t)=q^{\frac{i(3 i-1)}{2}} \sum_{s \geq|i|} q^{(s-i)(s+i)}\left[\begin{array}{c}
\left\lfloor\frac{n+i+1}{2}\right\rfloor \\
s+i
\end{array}\right]\left[\begin{array}{c}
\left\lfloor\frac{n-i}{2}\right\rfloor \\
s-i
\end{array}\right] t^{s},
$$

we have

$$
\sum_{i \in \mathbb{Z}}(-1)^{i} h_{i}(n, t)=\sum_{i=-n-1}^{n}(-1)^{i} h_{i}(n, t)=1 .
$$

By comparing coefficients we obtain the identity

$$
\begin{aligned}
& \sum_{i \in \mathbb{Z}}(-1)^{i} q^{\left(\begin{array}{c}
i \\
2
\end{array}\right)}\left[\begin{array}{c}
\left\lfloor\frac{n+i+1}{2}\right\rfloor \\
s+i
\end{array}\right]\left[\begin{array}{c}
\left\lfloor\frac{n-i}{2}\right\rfloor \\
s-i
\end{array}\right]= \\
& \sum_{i=-n-1}^{n}(-1)^{i} q^{\left(\begin{array}{c}
i \\
2
\end{array}\right)}\left[\begin{array}{c}
\left\lfloor\frac{n+i+1}{2}\right\rfloor \\
s+i
\end{array}\right]\left[\begin{array}{c}
\left\lfloor\frac{n-i}{2}\right\rfloor \\
s-i
\end{array}\right]=[s=0] .
\end{aligned}
$$

The last identity reduces to

$$
\sum_{i=-s}^{s}(-1)^{i} q^{\left(\begin{array}{c}
i \\
2
\end{array}\right)}\left[\begin{array}{c}
2 s \\
s-i
\end{array}\right]=[s=0]
$$

for $n \rightarrow \infty$.

\section{Remarks}

1) In order to obtain the first Rogers-Ramanujan identity (4.7) it suffices (cf. [2]) to prove the finite version only for even numbers $n$ and then letting $n \rightarrow \infty$. For the second identity we need only choose $n$ odd. P. Paule [14] has given a computer proof of these finite versions showing that both sides of the identities satisfy the same recursion, which in some sense trivializes the verification of the Rogers-Ramanujan identities.

2) Starting from (4.19) identity (4.4) can easily be derived by using Bailey's lemma (cf. [13]). By iterating this procedure one gets another finite version of the Rogers-Ramanujan identities. This approach is in some sense the opposite of our approach. 
3) Intimately connected with this Bailey chain approach is the following proof (cf. e.g. [16]):

Starting from(4.4), i.e. $\sum_{|i| \leq s}(-1)^{i} \frac{q^{\frac{i(3 i-1)}{2}}}{(q)_{s-i}(q)_{s+i}}=\frac{1}{(q)_{s}}$, and using (3.23) we get

$$
\begin{aligned}
& \sum_{s \geq 0} \frac{q^{s^{2}}}{(q)_{s}}=\sum_{s \geq 0} q^{s^{2}} \sum_{|i| \leq s}(-1)^{i} \frac{q^{\frac{i(3 i-1)}{2}}}{(q)_{s-i}(q)_{s+i}}= \\
& =\sum_{i \in \mathbb{Z}}(-1)^{i} q^{\frac{i(5 i-1)}{2}} \sum_{|i| \leq s} \frac{q^{s^{2}-i^{2}}}{(q)_{s-i}(q)_{s+i}}=\frac{1}{(q)_{\infty}} \sum_{i \in \mathbb{Z}}(-1)^{i} q^{\frac{i(5 i-1)}{2}} .
\end{aligned}
$$

Using (4.11) we get in the same way the second Rogers-Ramanujan identity. 


\section{References}

[1] A.K. Agarwal, D.M. Bressoud, Lattice paths and multiple basic hypergeometric series, Pac. J. Math. 136 (1989), 209-228

[2] G.E. Andrews, A polynomial identity which implies the RogersRamanujan identities, Scripta Math. 28 (1970), 297-305

[3] G. E. Andrews, The theory of partitions, Addison-Wesley 1976

[4] G. E. AndRews, On the proofs of the Rogers-Ramanujan identities, in $q$-Series and Partitions, The IMA volumes in Mathematics and its Applications 18 (1989), 1-14

[5] D. M. Bressoud, An easy proof of the Rogers-Ramanujan identities, J. Number Th. 16 (1983), 235-241

[6] D. M. Bressoud, Lattice paths and the Rogers-Ramanujan identities, Lecture Notes in Math. 1395 (1987), 140-172

[7] W. H. Burge, Restricted partition pairs, J. Comb.Th. A 63 (1993), $210-222$

[8] L. Carlitz, Fibonacci notes 4: q-Fibonacci polynomials, Fib. Quart. 13 (1975), 97-102

[9] J. Cigler, q-Fibonacci polynomials, Fib. Quart. 41 (2003), 31-40

[10] C. Krattenthaler, S.G. Mohanty, On lattice path counting by major and descents, Europ. J. Comb. 14 (1993), 43-51

[11] S.G. Mohanty, Lattice path counting and applications, Academic Press 1979

[12] M. Okado, A. Schilling, M. Shimozono, Crystal bases and qidentities, Contemp. Math. 291 (2001), 29-53

[13] P. Paule, The concept of Bailey chains, Sém. Lothar. Comb. B $18 \mathbf{f}$ (1987), 1-24 
[14] P. PAule, Short and easy computer proofs of the Rogers-Ramanujan identities and of identities of similar type, Electronic J. Comb. 1 (1994), $\mathrm{R} 10$

[15] H. Prodinger, Lecture notes on the course q-series in combinatorics and number theory,

http://www.wits.ac.za/helmut/postscriptfiles/q_course.ps.gz

[16] A. Schilling, S.O. WarnaAR, Conjugate Bailey pairs, Contemp. Math.297 (2002), 227-255

[17] A.V. Sills, Finite Rogers-Ramanujan type identities, Electronic J. Comb. 10 (2003), R 13

[18] I. ScHuR, Ein Beitrag zur additiven Zahlentheorie und zur Theorie der Kettenbrüche, 1917, in Gesammelte Abhandlungen, Bd.2, 117-136, Springer 1973 